\title{
Les défis de l'âge et de la santé des seniors
}

\author{
Jean Martin \\ Dr méd., membre de la rédaction
}

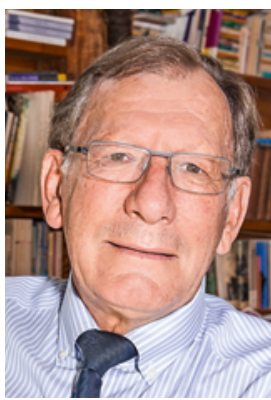

Pour son Assemblée des délégués de juin dernier, Curaviva, association des homes et institutions sociales suisses, a invité le philosophe et publiciste alémanique Ludwig Hasler à délivrer un «keynote speech», qu'il a intitulé "CURA c'est bien, VIVA c'est mieux". Nous vivons une époque formidable a-t-il dit, avec tout le potentiel de la digitalisation. Mais quelles seront demain les places respectives des hommes et des machines, quand nous aurons l'aide de robots "qui ne feront pas d'erreurs, ne seront jamais fatigués, jamais ivres, jamais amoureux" - et où il n'y aura plus d'accidents de la circulation parce que plus de chauffeurs humains...

Quel est le sens de l'âge dans une société devenue sans repères au plan métaphysique? Je suis issu, a dit Hasler, d'une famille de six enfants; après avoir accompagné notre maman et l'avoir vue s'affaiblir pour s'éteindre démente à 93 ans, notre appétit de vivre le plus longtemps possible a diminué. On sait que Google et les autres s'intéressent au transhumanisme, en direction de l'immortalité... mais en attendant l'assistance au suicide est plus en plus acceptée - et sollicitée. Dans un autre registre, nous devenons les comptables de notre état de santé. La prévention et la promotion de la santé sont évidemment de bonnes choses mais soyons attentifs à de possibles dérives hygiénistes.

Comme d'autres, l'orateur insiste: il s'agit de faire des patients, en particulier chroniques, des acteurs de leur propre santé. Schopenhauer: «Il n'y a pas de bonheur qui ne découle de l'utilisation de ses propres forces.» Métaphore théâtrale: «Tout le monde monte en scène, personne ne reste dans les coulisses». Le but (une évidence!) est de rendre ce qui reste à vivre digne d'être vécu; pour cela vouloir que, à chaque moment, les personnes âgées soient des sujets - aussi autonomes que possible malgré leurs limites fonctionnelles. Ne plus accepter leur «mise sous tutelle» dans la vie du home. Dans ce sens, les soignants/intervenants ont pour vocation d'être des animateurs, des revitalisateurs. Et il faut que le monde entre dans les institutions, l'air doit circuler. Interactions avec l'extérieur, avec des enfants, des animaux...
Un aspect très pratique est celui des risques que, avec le patient, on accepte de prendre dans la vie quotidienne. Une existence ultra-sécurisée inhibe, enferme, rend toujours plus dépendant. Les personnes ont le droit de prendre des risques, y compris de chute - cela leur laisse un libre arbitre. Se rappeler aussi les études qui montrent que le sourire et les échanges cordiaux ont, dans les institutions aussi bien sûr, un rôle promoteur de santé (salutogénétique) - humour aussi, être capable d'auto-dérision. "Il est essentiel de changer de paradigme», dit Hasler: injonction aujourd'hui fréquente. Une dimension de ce changement étant l'empowerment du patient, son implication dans les réflexions et décisions, y compris au plan médical et des soins.

Question: Cela est fort bien dit par un orateur charismatique, mais est-ce réaliste? Les services de santé et nos institutions (EMS, hôpitaux) ont-ils les ressources

\section{Quel est le sens de l'âge dans une société} devenue sans repères au plan métaphysique?

humaines et matérielles - et la volonté - de mettre en musique tous ces bons conseils? Les contraintes seront toujours présentes, cas échéant de plus en plus fermes. Pas sûr que les financeurs et politiques (eux qui pourtant ont des vies très pleines) soient très sensibles à la nécessité de mettre de la joie de vivre - quitte à prendre certains risques - dans la vie des seniors dépendants. Pas sûr que tous les soignants (au sens large) soient convaincus qu'il est bon de maximiser la possibilité pour les résidents de donner leur avis et de co-décider. Et cela demande du temps, un temps précieux! Dans tous les cas, il est toujours stimulant de bénéficier d'avis extérieurs, y compris profanes, de courants d'air par des gens qui pensent «hors du cadre», donnant des impulsions pour adapter les pratiques aux besoins. Revenant aux robots: que restera-t-il à faire de substantiel quand tant de tâches seront accomplies par des machines? Il restera à vivre, pleinement! Avec des soignants et accompagnants qui soient (devenus?), dit Hasler, des "spécialistes de la vie et de l'humain" - ce que à vrai dire beaucoup sont heureusement déjà. ${ }^{1}$ 